\title{
Hepatic atrophy treatment with portal vein embolization to control intrahepatic duct stenosis-associated cholangitis
}

\author{
Shin Hwang ${ }^{1}$, Gi-Young $\mathrm{Ko}^{2}$, and Dong-II Gwon ${ }^{2}$ \\ Departments of ${ }^{1}$ Surgery and ${ }^{2}$ Diagnostic Radiology, Asan Medical Center, \\ University of Ulsan College of Medicine, Seoul, Korea
}

\begin{abstract}
We present two cases of hepatic atrophy treatment with portal vein embolization (PVE) to control intractable cholangitis. The first case was a 60 -year-old male who was admitted for repeated episodes of cholangitis. He had undergone cholecystectomy and Roux-en-Y choledochojejunostomy 2 years earlier. Imaging studies showed left intrahepatic duct dilatation and anastomotic site stricture. The patient was reluctant to undergo another surgery. Thus, we decided to perform left PVE to induce atrophy of the left liver. The left liver shrank and stayed silent for 5 years, but a radiological intervention was necessary to treat symptomatic anastomotic stenosis. The patient has done well for 12 years after PVE. The second case was a 51-year-old female who was also admitted for repeated episodes of cholangitis. She had undergone excision of type I choledochal cyst 2 years earlier. Imaging studies showed right hepatic duct stenosis. Cholangitis developed repeatedly. Thus, radiologic interventions were performed 8 times over 9 years. Finally, she was referred for surgery, but she was very reluctant to undergo another surgery. We planned a wait-and-see strategy following right PVE. After PVE, the right liver progressively shrank. Three months after PVE, we decided to wait for a longer period until further atrophy of the right liver. The patient has been doing well for 14 months after PVE without any episode of cholangitis. In conclusion, experience from our two cases suggests that hepatic parenchymal induction therapy through percutaneous PVE can be a therapeutic option for patients with perihilar biliary stenosis-associated cholangitis. (Ann Hepatobiliary Pancreat Surg 2020;24:339-344)
\end{abstract}

Key Words: Biliary stenosis, cholangitis, liver atrophy; Portal vein embolization, radiological intervention

\section{INTRODUCTION}

Benign perihilar or intrahepatic biliary strictures inducing repeated episodes of cholangitis are difficult to treat through non-surgical methods. Thus, surgical treatment including resection of the involved liver portion is usually performed as the final definitive treatment after numerous repeated sessions of radiological intervention. The main mechanisms of cholangitis in patients with biliary stricture is stenosis-associated bile stasis, with or without combined ascending infection from patulous sphincter of Oddi or Roux-en-Y biliary-enteric anastomosis. If production of bile decreases with no change in biliary stenosis, bottlenecking bile stasis will be improved. Based on this theoretical basis, we have used a wait-and-see strategy after portal vein embolization (PVE) to control intrahepatic duct stenosis- associated cholangitis. ${ }^{1-7}$ We herein present two cases of hepatic atrophy treatment with PVE to control intractable cholangitis.

\section{CASE}

\section{Case 1}

The patient was a 60 -year-old male who was admitted for repeated episodes of cholangitis. He had undergone cholecystectomy and Roux-en-Y choledochojejunostomy 2 years earlier at an outside hospital probably because of iatrogenic bile duct injury during surgery for gallstone disease. Computed tomography (CT) showed left intrahepatic duct dilatation with abrupt luminal narrowing around the surgical anastomosis site and a suspicious high attenuating lesion in the left proximal intrahepatic duct,

Received: July 28, 2020; Revised: July 29, 2020; Accepted: July 29, 2020

Corresponding author: Shin Hwang

Department of Surgery, Asan Medical Center, University of Ulsan College of Medicine, 88 Olympic-ro 43-gil, Songpa-gu, Seoul 05505, Korea Tel: +82-2-3010-3930, Fax: +82-2-3010-6701, E-mail: shwang@amc.seoul.kr

Copyright (C) 2020 by The Korean Association of Hepato-Biliary-Pancreatic Surgery

This is an Open Access article distributed under the terms of the Creative Commons Attribution Non-Commercial License (http://creativecommons.org/ licenses/by-nc/4.0) which permits unrestricted non-commercial use, distribution, and reproduction in any medium, provided the original work is properly cited. Annals of Hepato-Biliary-Pancreatic Surgery - pISSN: 2508-5778 - elSSN: 2508-5859 

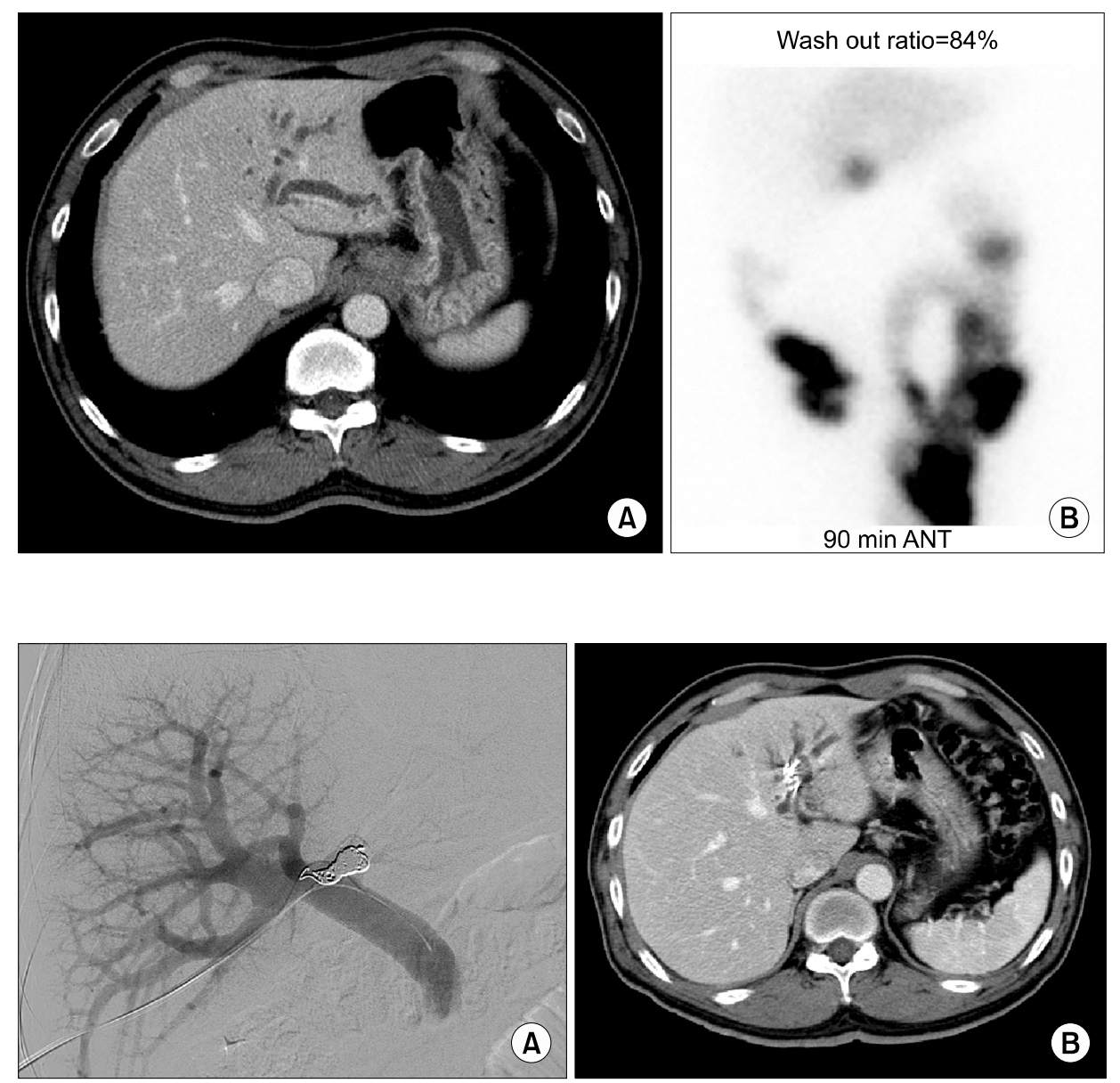

Fig. 2. Left portal vein embolization of the Case 1 (A) and computed tomography taken after 5 days (B).
Fig. 1. In image findings of the Case 1. (A) Computed tomography showed left intrahepatic duct dilatation with abrupt luminal narrowing around the surgical anastomosis site and suspicious high attenuating lesion in the left proximal intrahepatic duct. (B) Hepatobiliary scintigraphy showed delayed biliary excretion of the left liver.
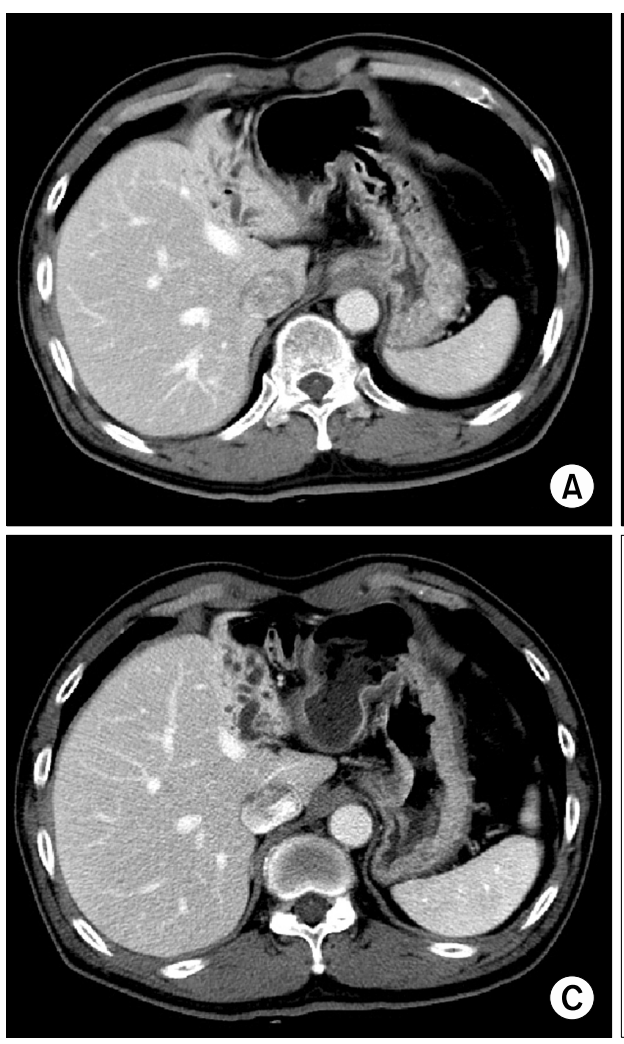

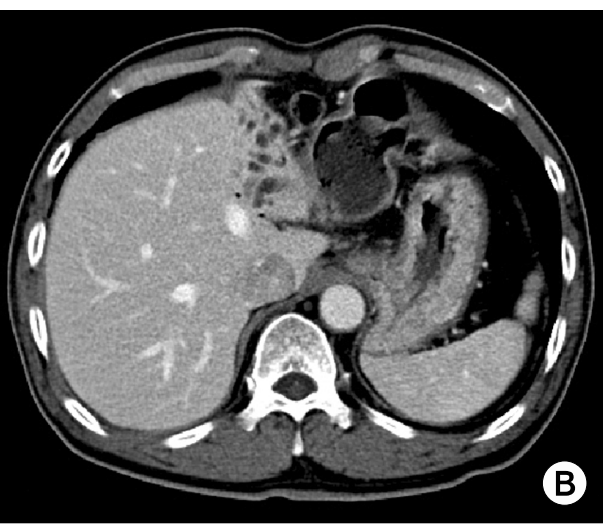

Wash out ratio $=94 \%$

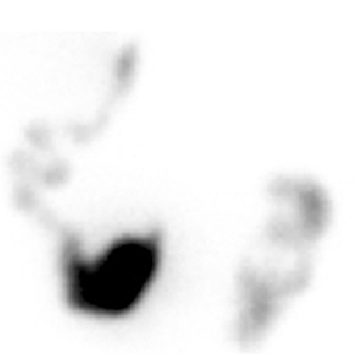

$90 \min$ ANT
Fig. 3. Follow-up computed tomography scan of the Case 1 at 7 days (A), 2 years (B) and 4 years $(C)$ after portal vein embolization. Hepatobiliary scintigraphy taken at 4 years showed normal biliary excretion (D). 
suggesting anastomotic site stricture (Fig. 1A). Hepatobiliary scintigraphy showed delayed biliary excretion of the left liver with normal excretion of the right liver (Fig.
1B). The patient was reluctant to undergo another surgery. Thus, we decided to perform left PVE to induce atrophy of the left liver (Fig. 2). We followed the patient with
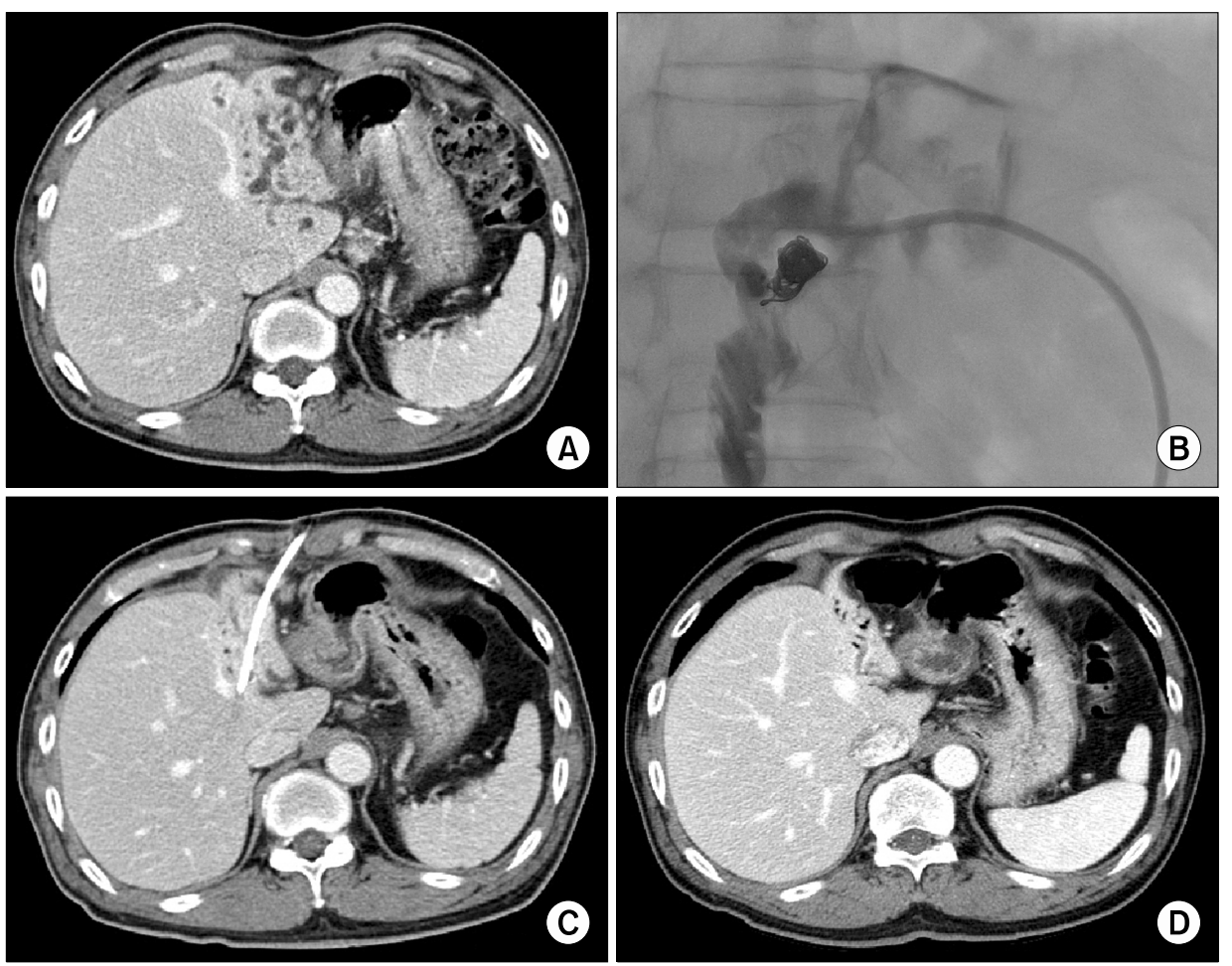

Fig. 4. Follow-up imaging studies of the Case 1 at 5 years after portal vein embolization. (A) The left hepatic ducts were dilated. (B) Percutaneous transhepatic biliary drainage was performed. (C) The left hepatic ducts were decompressed. (D) The left liver was shrunken.
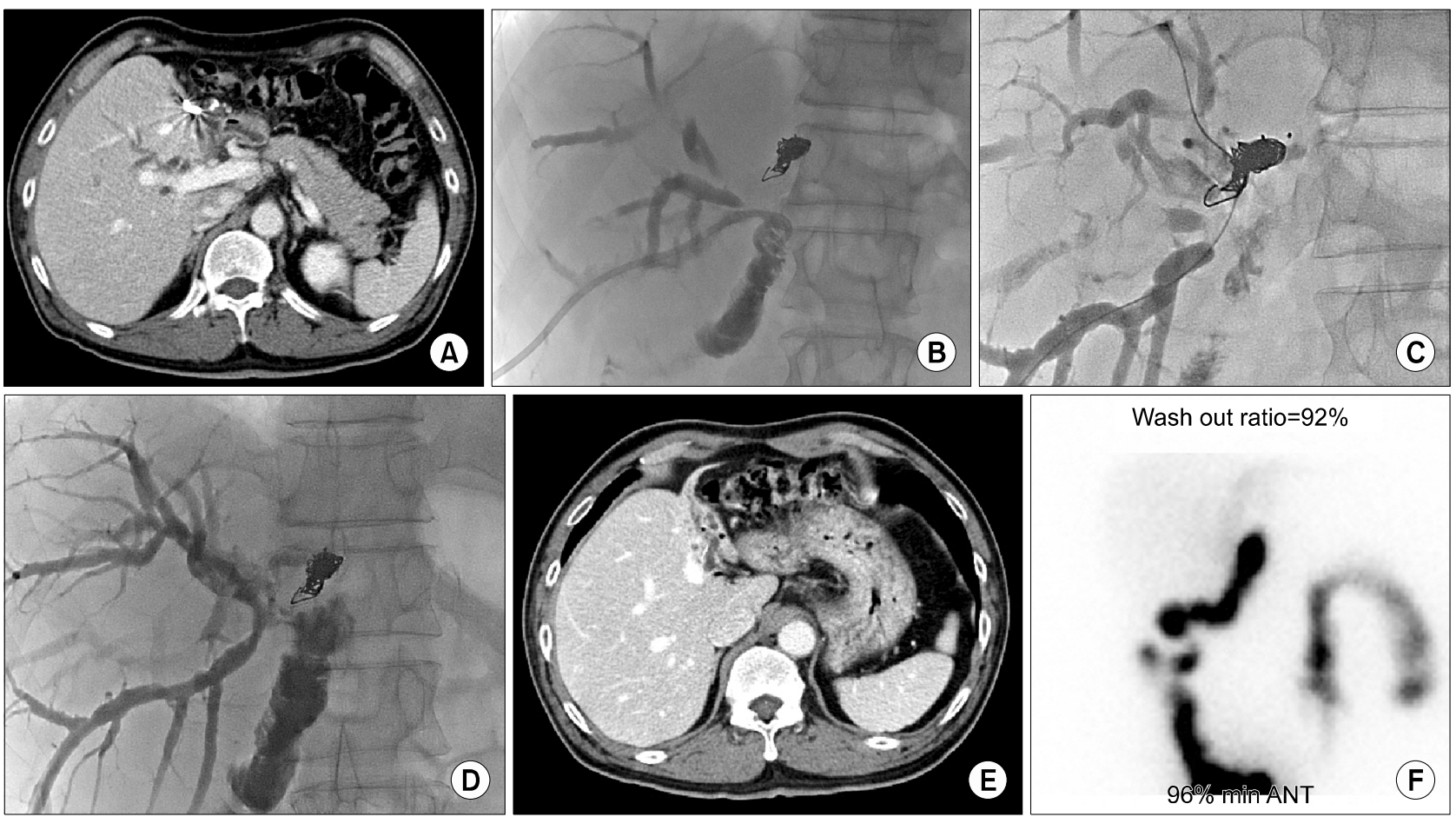

Fig. 5. Follow-up imaging studies of the Case 1 at 6 years after portal vein embolization. (A) There was cholangiohepatitis in the right liver and a $1 \mathrm{~cm}$-sized stone at the choledochojejunostomy site. (B-D) Percutaneous transhepatic biliary drainage and repeated balloon dilatation were performed. (E) Cholangitis was controlled. (F) Hepatobiliary scintigraphy showed normal biliary excretion. 
liver CTs every 3 months during the first year and yearly after that, in which the left liver was shrunken markedly without any episode of cholangitis (Fig. 3). However, after 5 years, cholangitis suddenly developed and CT scan showed development of multiple abscesses at the left lateral segment and caudate lobe. Thus, percutaneous transhepatic biliary drainage (PTBD) was performed through the left hepatic duct, and cholangitis was rapidly controlled (Fig. 4). After 6 years, CT scan showed cholangiohepatitis in the right liver and a $1 \mathrm{~cm}$-sized stone at the choledochojejunostomy site. Right PTBD was performed and repeated balloon dilatation was performed to the stenotic ducts and choledochojejunostomy site (Fig. 5). The PTBD tube was kept for 45 days. Thereafter, no episode of cholangitis developed. The patient has done well for 12 years after PVE.

\section{Case 2}

The patient was a 51-year-old female who was admitted for repeated episodes of cholangitis. She had undergone excision of type I choledochal cyst and Roux-en-Y hepaticojejunostomy 2 years earlier at an outside hospital (Fig. 6). CT scan and magnetic resonance cholangiography showed right intrahepatic duct stone with biliary dilatation (Fig. 7A, B). PTBD was performed and the stenotic sites were dilated. Intrahepatic stones were removed through percutaneous transhepatic cholangioscopy (Fig. 7C, D). PTBD and balloon dilatation were repeated 8 times over 9 years. Finally, she was referred for surgery, but she was very reluctant to undergo another surgery. We
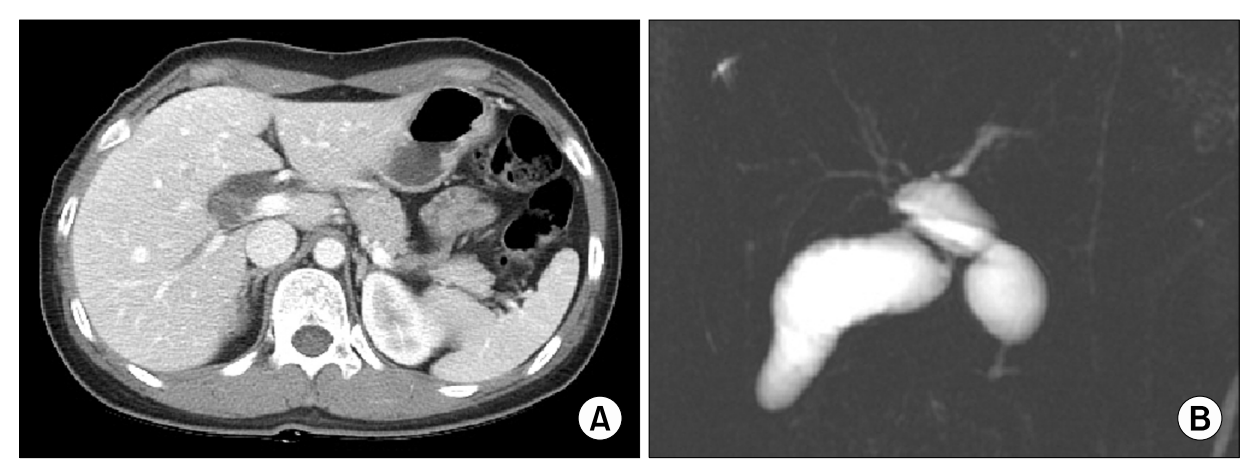

Fig. 6. Preoperative imaging studies of the Case 2 showing choledochal cyst of type I (A) and no marked dilatation of the intrahepatic ducts (B).
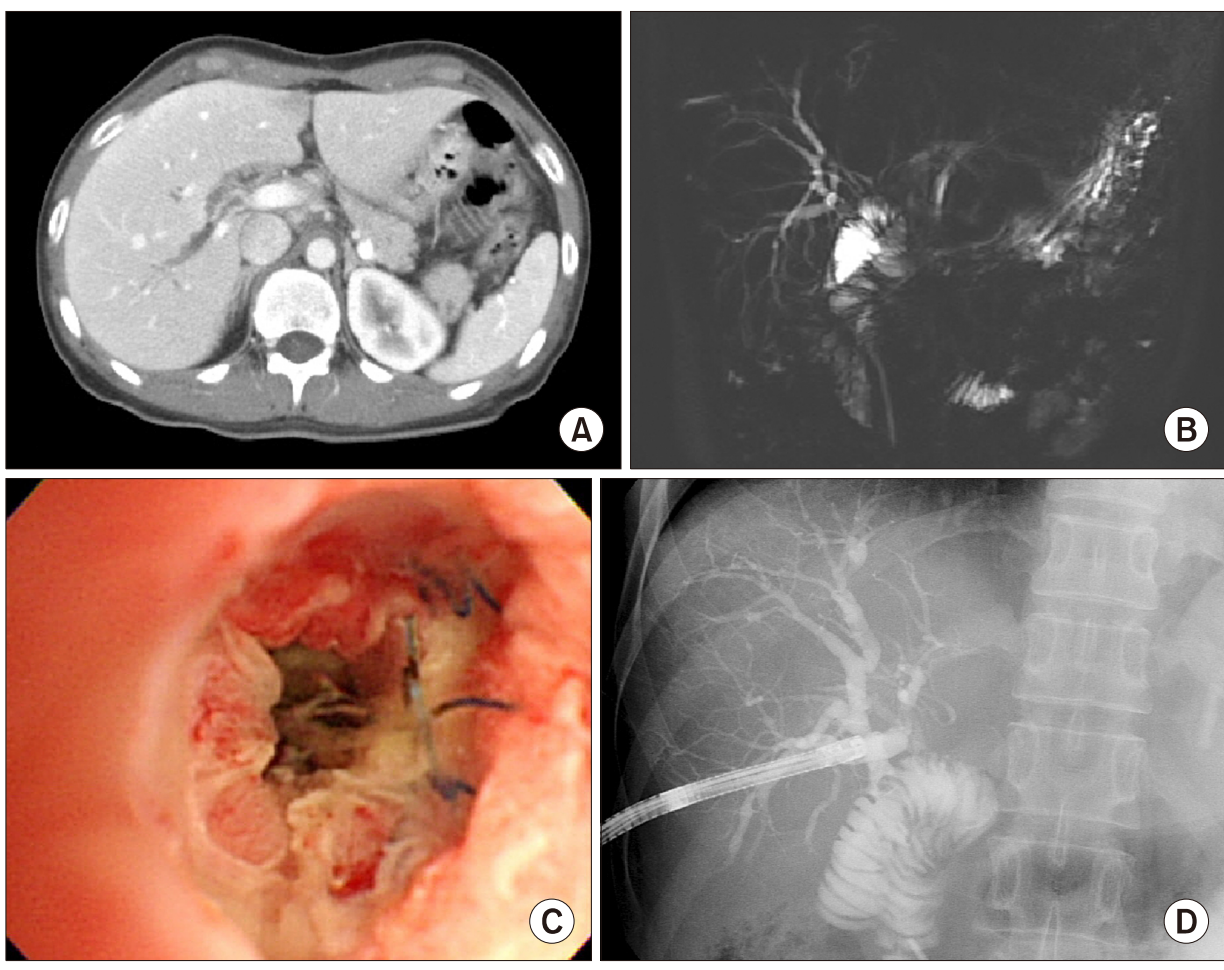

Fig. 7. Initial image findings of the Case 2. (A and B) Computed tomography and magnetic resonance cholangiography showed right intrahepatic duct stones with biliary dilatation. (C and D) Intrahepatic stones were removed through percutaneous transhepatic cholangioscopy. 

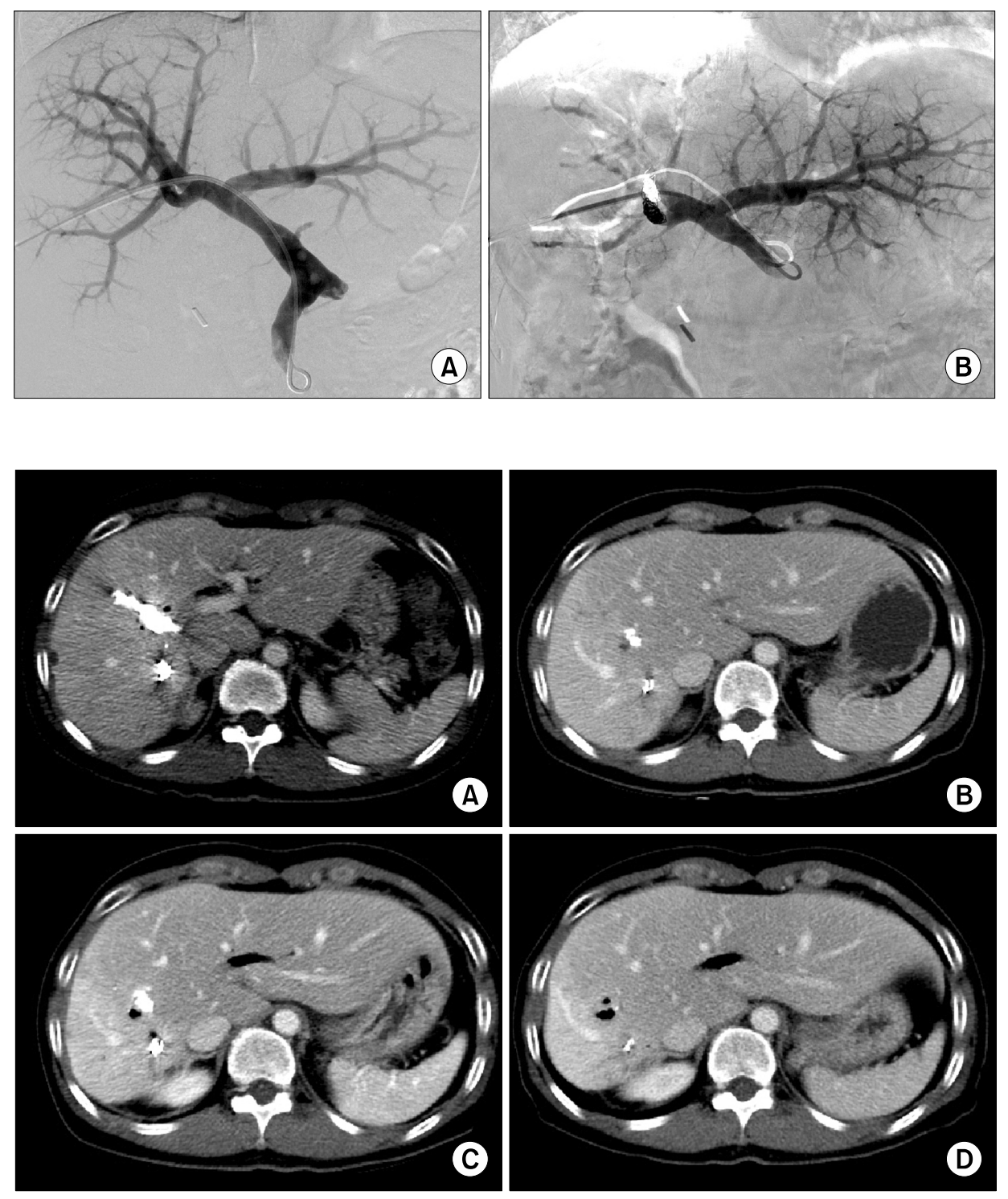

Fig. 8. Right portal vein embolization of the Case 2 before (A) and after (B) embolization.
Fig. 9. Follow-up computed tomography (CT) scans of the Case 2 during the first 1 year after portal vein embolization showing progressive atrophy of the right liver. CT scans were taken at 7 days (A), 1 month (B), 3 months (C) and 12 months (D) after portal vein embolization. explained the wait-and-see strategy after PVE and right PVE was performed (Fig. 8). After PVE, the right liver progressively shrank (Fig. 9). Three months after PVE, we decided to wait for a longer period until further atrophy of the right liver. Twelve months after PVE, we decided to perform regular follow-ups with imaging studies unless cholangitis developed again. The patient has done well for 14 months after PVE without any episode of cholangitis.

\section{DISCUSSION}

Deprivation of portal blood flow induces atrophy of the ipsilateral liver. ${ }^{8,9}$ We previously reported the effectiveness and usefulness of hepatic atrophy induction treatment for isolated segmental bile duct injury following laparo- scopic cholecystectomy and central hepatectomy. ${ }^{1-3}$

In our previous cases, ${ }^{1-3}$ bile leak was present. Thus, the management process was complex and close observation was necessary. In patients with bile duct injury, two mechanisms are associated with hepatic parenchymal atrophy, deprivation of portal blood flow and bile duct obstruction. The treatment procedure consisted of three major steps. The first step was embolization of the segmental portal branch, which effectively inhibited the quantity and quality of bile production. ${ }^{7,10}$ The second step was the closure of the leakage site through the induction of heavy adhesion to ensure clamping of the PTBD or pigtail drainage. The third step was the inhibition of bile drainage through spontaneous occlusion of the segmental duct to accelerate atrophy of the segmental parenchyma. These 
three-step procedures usually took 3-4 months. ${ }^{1}$

Unlike the abovementioned cases, our present patients did not have bile leaks, so only wait-and-see approach was necessary following PVE. The speed of hepatic parenchymal atrophy varies depending on the status of the liver and embolic methods and materials for PVE. Therefore, the concepts of kinetic growth and shrinkage rates are used. ${ }^{5,9,10}$ These rates are high in the order of normal, chronic hepatitis and cirrhotic livers. The left liver in our first case was compatible with a chronic hepatitis liver because there were significant precedent injuries inducing fibrosis. In contrast, the second case liver was compatible with a normal liver because the right liver parenchyma was well-preserved despite repeated episodes of cholangitis. If the right liver did not shrink significantly 1-2 months after right PVE, our choice would have been upfront surgery or further waiting after additional right hepatic vein embolization.

Intrahepatic stone formation is an indication for surgery instead of atrophy induction unless the stones are removed completely. Such formation of intrahepatic stones indicates the presence of severe stenosis and repeated episodes of inflammation, which can be risk factors for malignant transformation. Our two patients also had risks for late malignant transformation at the intrahepatic bile ducts of the shrunken liver, thus we suggest that life-long surveillance is necessary for such patients with hepatolithiasis or parenchymal atrophy. ${ }^{11}$

In conclusion, the experience from our two cases and other precedent cases suggests that hepatic parenchymal induction therapy through percutaneous PVE can be a therapeutic option for patients with perihilar biliary stenosis-associated cholangitis.

\section{ORCID}

Shin Hwang: https://orcid.org/0000-0002-9045-2531
Gi-Young Ko: https://orcid.org/0000-0003-4617-1799

Dong-Il Gwon: https://orcid.org/0000-0002-3121-2853

\section{REFERENCES}

1. Hwang S, Yoon SY, Jung SW, Namgoong JM, Park GC, Gwon DI, et al. Therapeutic induction of hepatic atrophy for isolated injury of the right posterior sectoral duct following laparoscopic cholecystectomy. Korean J Hepatobiliary Pancreat Surg 2011;15: 189-193.

2. Hwang S, Lee SG, Lee YJ, Ha TY, Ko GY, Song GW. Delayed-onset isolated injury of the right posterior segment duct after laparoscopic cholecystectomy: a report of hepatic segmental atrophy induction. Surg Laparosc Endosc Percutan Tech 2007; 17:203-205.

3. Hwang S, Park GC, Ha TY, Ko GY, Gwon DI, Choi YI, et al. Hepatic parenchymal atrophy induction for intractable segmental bile duct injury after liver resection. Hepatogastroenterology 2012; 59:866-868.

4. Hwang S, Ha TY, Ko GY, Kwon DI, Song GW, Jung DH, et al. Preoperative sequential portal and hepatic vein embolization in patients with hepatobiliary malignancy. World J Surg 2015; 39:2990-2998.

5. Hwang S, Ko GY, Kim MH, Lee SK, Gwon DI, Ha TY, et al. Preoperative left portal vein embolization for left liver resection in high-risk hepatobiliary malignancy patients. World J Surg 2016; 40:2758-2765.

6. Ko GY, Hwang S, Sung KB, Gwon DI, Lee SG. Interventional oncology: new options for interstitial treatments and intravascular approaches: right hepatic vein embolization after right portal vein embolization for inducing hypertrophy of the future liver remnant. J Hepatobiliary Pancreat Sci 2010;17:410-412.

7. Hwang S, Lee SG, Ko GY, Kim BS, Sung KB, Kim MH, et al. Sequential preoperative ipsilateral hepatic vein embolization after portal vein embolization to induce further liver regeneration in patients with hepatobiliary malignancy. Ann Surg 2009;249: 608-616.

8. Yokoyama Y, Nagino M, Nimura Y. Mechanisms of hepatic regeneration following portal vein embolization and partial hepatectomy: a review. World J Surg 2007;31:367-374.

9. Shindoh J, Truty MJ, Aloia TA, Curley SA, Zimmitti G, Huang $\mathrm{SY}$, et al. Kinetic growth rate after portal vein embolization predicts posthepatectomy outcomes: toward zero liver-related mortality in patients with colorectal liver metastases and small future liver remnant. J Am Coll Surg 2013;216:201-209.

10. Uesaka K, Nimura Y, Nagino M. Changes in hepatic lobar function after right portal vein embolization. An appraisal by biliary indocyanine green excretion. Ann Surg 1996;223:77-83.

11. Hwang S, Lee SG, Lee YJ, Park KM, Choi DR, Kim KH, et al. Right hepatic lobectomy in the treatment of right-sided hepatolithiasis. J Korean Surg Soc 1999;57:550-557. 